\title{
Formation of Gold Nanorods by A Stochastic
}

\section{'Popcorn’ Mechanism}

Jonathan A. Edgar, Andrew M. McDonagh, and Michael B. Cortie*

Institute for Nanoscale Technology, University of Technology Sydney, PO Box 123, Broadway, NSW 2007, Australia

\section{Table of Contents synopsis and graphic}

The formation of gold nanorods by the seeded wet-chemical process proceeds by a stochastic, 'popcorn'-like mechanism, in which individual seeds lie quiescent for some time before suddenly and rapidly growing into rods. This is quite different from the steady, concurrent growth of nanorods that has been previously generally assumed.

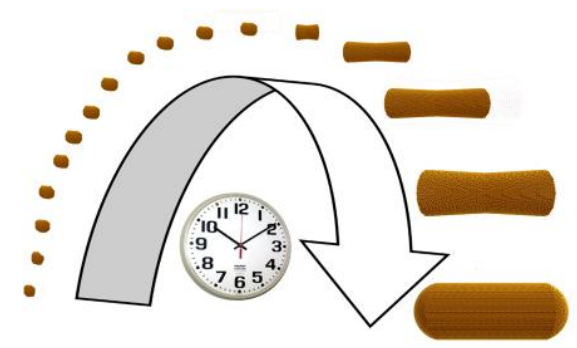




\section{Formation of Gold Nanorods by A Stochastic 'Popcorn' Mechanism}

Jonathan A. Edgar, Andrew M. McDonagh, and Michael B. Cortie*

Institute for Nanoscale Technology, University of Technology Sydney, PO Box 123,

Broadway, NSW 2007, Australia

\section{RECEIVED DATE}

TITLE RUNNING HEAD Nucleation of gold nanorods.

CORRESPONDING AUTHOR FOOTNOTE Prof. M Cortie, email:

michael.cortie@uts.edu.au,

Phone: (+61)-2-9514-2208 


\begin{abstract}
Gold nanorods have significant technological potential and are of broad interest to the nanotechnology community. The discovery of the seeded, wet-chemical synthetic process to produce them may be regarded as a landmark in the control of metal nanoparticle shape. However, the mechanism by which the initial spherical gold seeds acquire anisotropy is a critical, yet poorly understood, factor. Here we examine the very early stages of rod growth using a combination of techniques including cryogenic transmission electron microscopy, optical spectroscopy and computational modeling. Reconciliation of the available experimental observations can only be achieved by invoking a stochastic, 'popcorn'-like mechanism of growth, in which individual seeds lie quiescent for some time before suddenly and rapidly growing into rods. This is quite different from the steady, concurrent growth of nanorods that has been previously generally assumed. Furthermore we propose that the shape is controlled by the ratio of surface energy of rod sides to rod ends, with values of this quantity in the range of 0.3 to 0.8 indicated for typical growth solutions.
\end{abstract}

KEYWORDS gold nanorods, shape, kinetics, mechanism, cryo-TEM, modeling 
$\mathrm{G}$ old nanorods have strongly anisotropic optical properties, which suggest diverse technological applications. ${ }^{1-5}$ The rods exhibit optical absorption peaks arising from surface plasmon resonances in the longitudinal as well as transverse directions. The resonances can be tuned to absorb light with wavelengths ranging from 520 to more than $1600 \mathrm{~nm}$ by control of the aspect ratio (the length / diameter ratio) of the rods. ${ }^{6-8}$ Although gold nanoshells possess similar tunability and absorption efficiencies, ${ }^{9-11}$ gold nanorods arguably offer a more convenient platform ${ }^{12-13}$ because they are more readily synthesized. The 'seeded process ${ }^{14}$ (Equ. 1) to grow nanorods is now widely adopted:

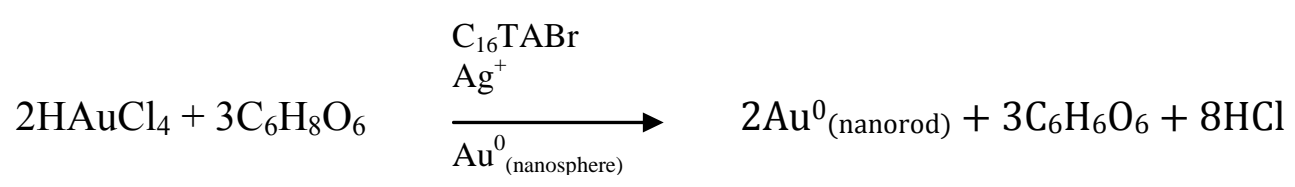

where $\mathrm{C}_{6} \mathrm{H}_{8} \mathrm{O}_{6}$ is ascorbic acid and $\mathrm{C}_{6} \mathrm{H}_{6} \mathrm{O}_{6}$ is dehydroascorbic acid (DHA), the oxidized form of ascorbic acid. Silver nitrate is usually added to provide shape control $\left(\left[\mathrm{C}_{16} \mathrm{TA}\right][\mathrm{AgBr}]\right.$ has also been used ${ }^{15}$ (where $\mathrm{C}_{16} \mathrm{TA}=$ cetyltrimethylammonium)). Related routes requiring neither pre-synthesized seed ${ }^{15-17}$ or $\mathrm{Ag}^{18-19}$ have also been demonstrated.

In the 'seeded' process, gold nanorods are formed by the addition of small gold nanoparticle seeds (usually $\sim 1$ to $3 \mathrm{~nm}$ in diameter) to an aqueous 'growth solution' consisting of a mixture of surfactant $\left(\mathrm{C}_{16} \mathrm{TABr}\right.$ ), ionic gold (usually $\left.\mathrm{AuCl}_{4}{ }^{-}\right)$, weak reductant (ascorbic acid) and ionic silver. The resultant nanorods are usually $10-20 \mathrm{~nm}$ in diameter and $30-60 \mathrm{~nm}$ in length, and the dimensions are typically determined by the amount of seed and/or by the ionic silver concentration. If the concentration of $\mathrm{C}_{16} \mathrm{TABr}$ is at least tenfold greater than that of $\mathrm{AuCl}_{4}^{-}$, then the gold complex is solubilized within the surfactant micelles. ${ }^{7} \mathrm{C}_{16} \mathrm{TABr}$ has an aggregation number of around $100-200$ (depending on other solution components), ${ }^{20}$ and a size of about 2.5 by $4 \mathrm{~nm},{ }^{21}$ so that, in the range of concentrations employed in most nanorod syntheses, there may on average be only one or 
two gold-containing ions associated with a micelle. Although chloride ions may poison rodgrowth reactions, ${ }^{19,22-23}$ the concentration arising from those released from $\mathrm{AuCl}_{4}{ }^{-}$has an insignificant effect in this case.

There is still difficulty in explaining how the initially isotropic seed particle switches to an anisotropic nanorod geometry, particularly in the case of silver-assisted synthesis which produces nanorods that are single crystals. ${ }^{24}$ To start with, these particles possess six equivalent growth directions. Somehow, only two of these, at $180^{\circ}$ to one another, are selected in the transition from seed to rod. In syntheses performed without silver ions, the resulting nanorods are pentagonally twinned and it has been suggested that the anisotropic factor that seeds rod growth may be twinning in the seed. ${ }^{7}$ Alternately, it has been proposed for syntheses with or without silver ions that electrostatic considerations were responsible, with rod growth favored at sites of greater surface curvature. ${ }^{19}$ However, this still leaves the question of how an anisotropic particle with a region of greater curvature was established in the first place.

Attempts have been made to resolve this puzzle by determining the particle geometry at various stages in the growth process. ${ }^{25-27}$ For example, arresting the nanorod growth at various times by additions of sulfide ions has been investigated, ${ }^{26}$ but there is no guarantee that chemical and subsequent centrifugation treatments do not affect the particle geometry in this case. Another method to determine the dimensions of nanorods is to match the kinetically monitored longitudinal peak position to a range of simulated optical spectra. ${ }^{28-29}$ This method requires detailed knowledge of the actual geometry of the nanorods because particles with equal aspect ratios but varied geometries e.g. ellipsoids or sphero-cylinders, will display different spectra; additionally, simply changing the end-cap geometry can cause a significant peak shift. ${ }^{30-31}$ Henkel et al. and Morita et al. used SAXS to estimate the in situ particle 
dimensions of growing nanorods, ${ }^{32-33}$ and then invoked the optical determination of Brioude et $a l .{ }^{34}$ to validate their results in terms of the aspect ratio.

In general, however, detailed characterization of the very earliest stages of growth, in which the equiaxed seeds begin to grow anisotropically, has eluded investigators. This is the topic that we address here.

The directed growth of gold nanorods is generally attributed to the preferential passivation of the sides of a growing rod by the formation of a $\mathrm{C}_{16} \mathrm{TABr}$ bilayer, ${ }^{35-36}$ leaving the ends of the rod free to grow, however, there has also been a suggestion that the mechanism involves soft templating by $\mathrm{C}_{16} \mathrm{TABr}$ micelles. ${ }^{16,36}$ If $\mathrm{Ag}$ is present, it has been determined to be on the sides of the nanorods in the form of a sparingly soluble Ag-containing complex with the formula $\mathrm{C}_{19} \mathrm{H}_{42}-\mathrm{NAgBr}_{2} .{ }^{15,37}$ The sides of the rods generally present relatively loosely packed crystallographic planes to the environment, e.g. $\{110\}^{24}$ or higher index (e.g. $\{250\}^{38}$ ) planes, and it has also been suggested that underpotential deposition (UPD) of $\mathrm{Ag}^{0}$ on such loosely packed $\mathrm{Au}$ facets is responsible for their passivation. ${ }^{24}$ However, due to the high concentration of bromide ions in the growth solution, it might be expected that the silver metal will return to the complex form. A mechanism where $\mathrm{AgBr}$ (rather than $\mathrm{Ag}^{0}$ ) shields the sides of the nanorod to promote elongation has also been mooted. ${ }^{14-15,36,39}$ The net effect of any of these processes is to retard inwards mass transfer of $\mathrm{Au}$ onto the sides of the nanorods, thus encouraging anisotropic growth at the ends. It is now well known that $\mathrm{C}_{16} \mathrm{TABr}$ is considerably superior to any of the other single chain cationic surfactants in regard to growth of gold nanorods. ${ }^{19,22}$ The reasons appear to be that (1) the $\mathrm{C}_{16}$ tail has the optimum steric properties and aqueous solubility to form a stable bilayer ${ }^{19,22}$ and (2) of several known counter-ions for the $\mathrm{C}_{16} \mathrm{TA}^{+}, \mathrm{Br}^{-}$provides a salt with the strongest adherence to an $\mathrm{Au}$ surface. ${ }^{40}$ 
From previous studies examining the growth rates of nanorods, ${ }^{17,} 29,32-33$ it was reported that the radial and longitudinal growth rates are initially comparatively rapid, and then decline asymptotically to zero. A coupled pair of empirical growth models for Au nanorods has been proposed. ${ }^{32}$ These can certainly be used to empirically reproduce the appearance and observed shifts of the longitudinal plasmon peak (see Supporting Information). However, a critical assumption in this approach and in most previous studies, is that all seed particles grow to rods simultaneously. Nevertheless, it may be deduced from the details of the multistep rod growth protocol of Busbee et al., for example, that growth times of 3 to 5 seconds are already sufficient to convert at least some seeds to rods. ${ }^{18}$ Here we show that the assumption of parallel growth is generally false, even in the single-stage technique of rod growth. The transition of seed particles to rods actually takes place by a stochastic process so that there is a period in which significant proportions of seed nuclei and growing rods of various shapes and sizes co-exist. This is a very different situation from that usually assumed for nucleation and growth of colloidal nanoparticles.

\section{RESULTS}

In this section, we first discuss the chemistry involved in the formation of gold nanorods followed by our investigations into their anisotropic growth. The important chemical reactions involved in the synthesis of gold nanorods are summarized in Scheme 1. Upon addition of $\left[\mathrm{Au}^{\mathrm{III}} \mathrm{Cl}_{4}\right]^{-}$to a $\mathrm{C}_{16} \mathrm{TABr}$ solution, we (see Section 3 of Supporting Information) and others ${ }^{41-43}$ have identified the Au species present in micellar $\mathrm{C}_{16} \mathrm{TABr}$ solution as $\mathrm{AuBr}_{4}{ }^{-}$, with the bromide ions originating from the partially dissociated $\mathrm{C}_{16} \mathrm{TABr}^{44-45}$ Once dispersed in the micellar $\mathrm{C}_{16} \mathrm{TABr}$ solution, $\mathrm{Au}^{\mathrm{III}} \mathrm{Br}_{4}{ }^{-}$is reduced in a two-electron process to $\mathrm{Au}^{\mathrm{I}} \mathrm{Br}_{2}{ }^{-}$by the addition of ascorbic acid (reportedly via an inner-sphere process involving a $\mathrm{Au}^{\mathrm{III}}$ ascorbate complex). ${ }^{46}$ The ascorbic acid is itself oxidized to dehydroascorbic acid (also a 
two-electron process). It is possible for the oxidation products of ascorbic acid, dehydroascorbic and 2,3 - diketo - gulonic acids, to reduce gold ions ${ }^{47}$ but elevated temperatures (approaching $100^{\circ} \mathrm{C}$ ) are typically required before this reduction is evident on a short time scale.

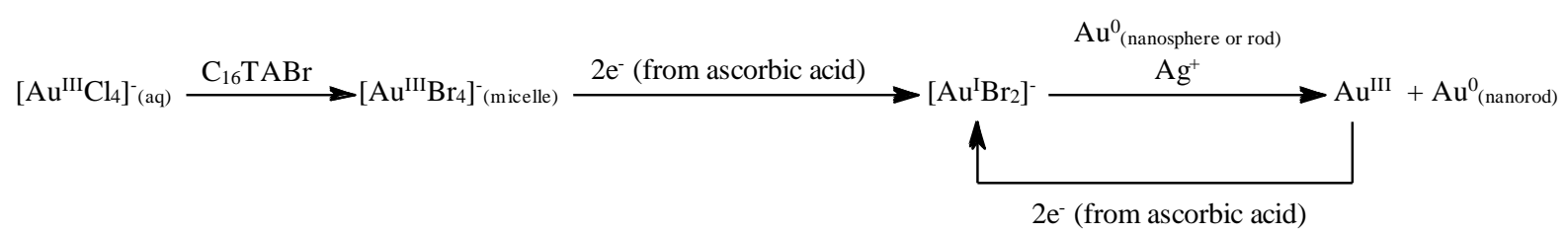

Scheme 1: Reactions involved in the surfactant-mediated growth of gold nanorods.

Ascorbic acid in $\mathrm{C}_{16} \mathrm{TABr}$ solution dissociates to a greater extent than in water alone indicating an interaction with the surfactant monomers or micelles ${ }^{48}$ and the oxidation potential of ascorbic acid is lower in micellar environments ${ }^{46}$ than in pure aqueous solutions. Importantly however, at this stage we find that $\mathrm{Au}^{\mathrm{I}}$ ions in the growth solution are not reduced to $\mathrm{Au}^{0}$ at any appreciable rate even when an excess of up to five molar equivalents of ascorbic acid is present. $\mathrm{Au}^{\mathrm{I}}$ ions in the growth solution are reduced to $\mathrm{Au}^{0}$ only upon addition of the Au nanoparticle seeds.

Figure 1 presents data obtained from UV-visible spectroscopy experiments that show the amount of $\mathrm{Au}^{0}$ after nanorod growth has completed, as a function of the amount of added ascorbic acid (see Section 4 of Supporting Information for complete data). No $\mathrm{Au}^{0}$ is produced at stoichiometries of less than $1: 1$ ascorbic acid : $A u^{\text {III }}$. In this range, the additions of ascorbic acid reduce $\mathrm{Au}^{\mathrm{III}}$ to $\mathrm{Au}^{\mathrm{I}}$ only. However, after an equimolar amount of ascorbic acid has been added, $\mathrm{Au}^{0}$ is produced in an approximately 2:1 proportion to the further additions of ascorbic acid within the growth solution. This is in agreement with the results predicted by stoichiometry. 


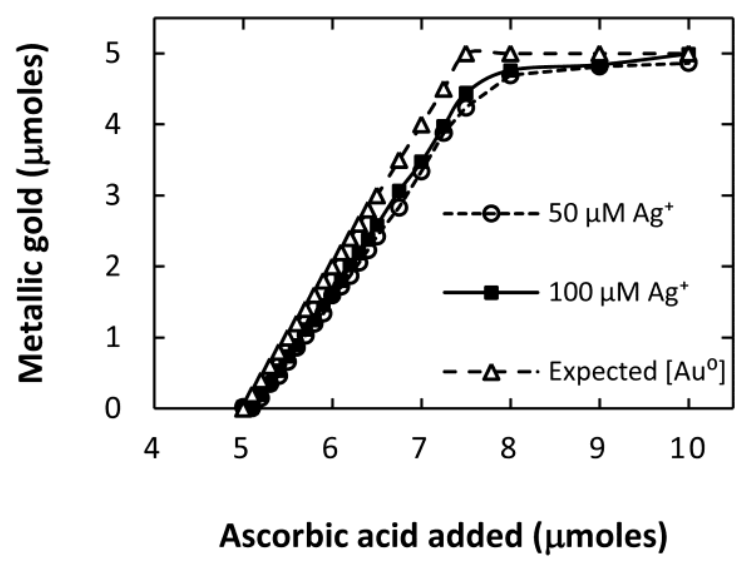

Figure 1. Graph showing the amount of $\mathrm{Au}^{0}$ formed as a function of added ascorbic acid in a mixture comprised of growth solution and seed. The initial gold content for all experiments is $5 \mu$ moles of $\mathrm{Au}^{\mathrm{III}}$.

A key feature of the disproportionation of $\mathrm{Au}^{\mathrm{I}}$ via $3 \mathrm{Au}^{\mathrm{I}} \rightarrow 2 \mathrm{Au}^{0}+\mathrm{Au}^{\mathrm{III}}$ that helps to establish its role during the formation of nanorods is that the disproportionation reaction is (1) catalyzed by metallic gold ${ }^{49-50}$ and is (2) therefore autocatalytic. ${ }^{14}$ As mentioned above, we found that no reaction occurred in the absence of gold seed, even when the concentration of ascorbic acid was increased to five times that required, but the reaction proceeded rapidly when $\mathrm{Au}^{0}$ particles were added, thus verifying (1). Figure 2 shows the amount of $\mathrm{Au}^{\mathrm{I}}$ that has been reduced to the theoretical maximum amount of $\mathrm{Au}^{0}$ obtainable (with ascorbic acid the limiting reactant) during the reaction with growth solution. The sigmoidal shape of the curve indicates that the reaction is autocatalytic, ${ }^{17}$ thus verifying (2). For the reaction to proceed to completion, any $\mathrm{Au}^{\mathrm{III}}$ formed by disproportionation must be rapidly reduced back to $\mathrm{Au}{ }^{\mathrm{I}}$ by excess ascorbic acid to avoid it re-oxidizing any $\mathrm{Au}^{0}{ }^{19}, 51$ However, from a purely stoichiometric perspective it does not matter whether the $\mathrm{Au}^{0}$ is produced by disproportionation of $\mathrm{Au}^{\mathrm{I}}$ or by direct reduction of $\mathrm{Au}^{\mathrm{I}}$ by ascorbic acid because both processes still require one mole of ascorbic acid per two moles of $\mathrm{Au}^{0}$ produced from $\mathrm{Au}$. 
Based on our data, the sequence of reactions that we propose occurs on the surface of a growing gold nanoparticle are shown schematically in Figure 3.

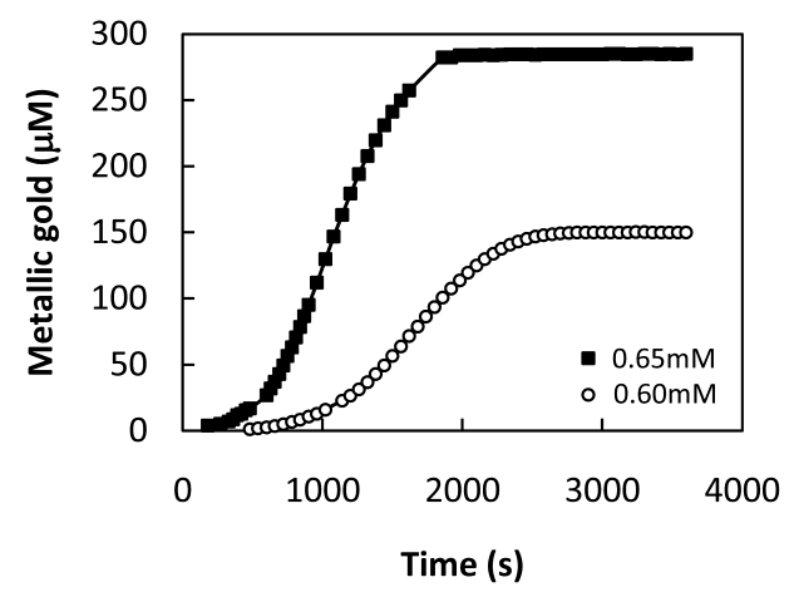

Figure 2. Graph showing the concentration of $\mathrm{Au}^{0}$ (as suspended nanoparticles) resulting from the reaction of two different concentrations of ascorbic acid with $\mathrm{Au}$.

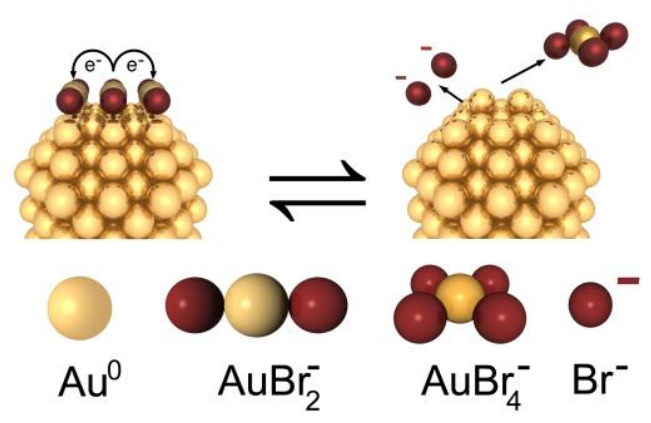

Figure 3. Disproportionation of $\mathrm{AuBr}_{2}{ }^{-}$according to the reaction $3 \mathrm{Au}^{\mathrm{I}} \rightarrow 2 \mathrm{Au}^{0}+\mathrm{Au}^{\mathrm{III}}$ at the surface of a $2 \mathrm{~nm}$ diameter gold nanoparticle leading to growth of the particle (approximately to scale).

Figure 4 shows UV-visible spectra recorded during the growth of nanorods in solution, in this case produced with a growth solution containing $0.50 \mathrm{mM} \mathrm{HAuCl}_{4}, 0.1 \mathrm{M} \mathrm{CTABr}, 0.65$ $\mathrm{mM}$ ascorbic acid and $70 \mu \mathrm{M} \mathrm{AgNO}_{3}$. A longitudinal plasmon resonance at $\sim 750 \mathrm{~nm}$ appears in the very earliest stages of growth $(t<180 \mathrm{~s})$. The peak red-shifts to $\sim 800 \mathrm{~nm}$ over a period of $\sim 180 \mathrm{~s}$ and subsequently blue-shifts to $\sim 660 \mathrm{~nm}$, a trend also reported by others. ${ }^{25,27,31,52-}$ 55 These data confirm that rods can form quite early in the synthetic process and that the 
shape then changes during the growth phase. However, the conventional interpretation of such early spectra would be that they had been generated by an ensemble of tiny rods, each well below the final rod size. Tantalizing evidence to the contrary is available from experimental data provided in the prior literature but the significance was evidently not fully recognized at the time. For example, TEM images provided by Sau and Murphy, ${ }^{25}$ and Niidome $e t a l .{ }^{27}$ show that nanorods of nearly full size were present even at the earliest stages of the synthesis process, at a time when the absorbance of the colloid was still low, while Miranda et al. interpreted the blue shift of the longitudinal resonance as evidence that nucleation and growth had operated in a parallel rather than sequential fashion within their photochemically-activated growth solution. ${ }^{56}$

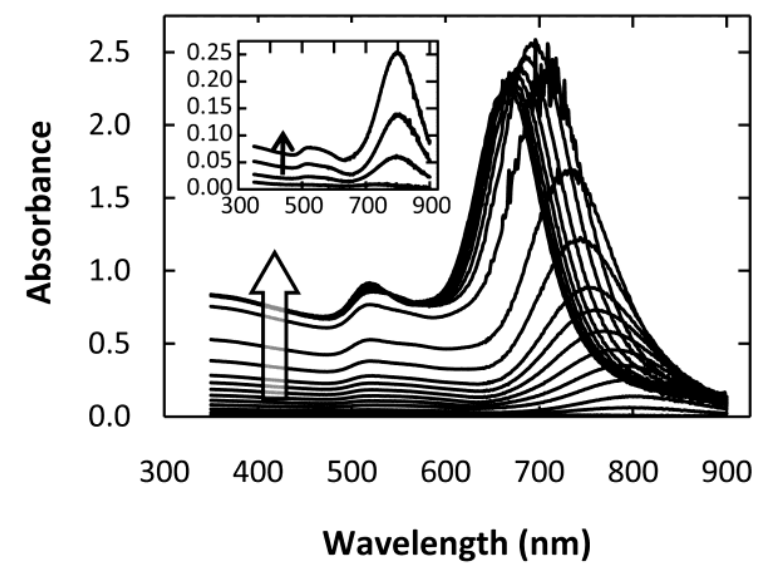

Figure 4. Representative spectra of the kinetics of gold nanorods in solution. Inset shows an enlargement of the data recorded up to 600 seconds. The earliest trace shown in either panel is for 2.5 minutes.

To further investigate the rapid growth phase, cryogenic transmission electron microscope ('cryo-TEM') images of rapidly frozen solutions containing nanorods were obtained. Figure 5 shows images obtained after 2.5 minutes and up to 24 hours after the addition of seed particles to growth solutions. Surprisingly, rods of nearly full size are evident after only 2.5 
minutes into the growth phase. At the synthesis concentrations used and considering the observed particle dimensions, the nanorod concentration is on the order of nanomolar and hence rods are only sparsely distributed in the TEM samples. Nevertheless, the present work was sufficiently exhaustive that we conclude that rods of sizes between those shown and that of the starting seed must be exceedingly rare. Other workers who have provided TEM images of the earliest stages of rod growth ${ }^{24-25}$ have also not reported rods smaller than those shown here. Even though a much more exhaustive TEM study may find examples of even smaller rods, the implication of the present results is that rods must pass through this size range very rapidly indeed. In addition it is clear that a seed can transition into a rod of nearly full size in about two minutes. We note that the cryogenic process used to produce the TEM samples would have rapidly halted the growth of the nanorods once freezing had occurred and so we conclude that if any very small rods had been present in the examined material they would have been preserved and found.

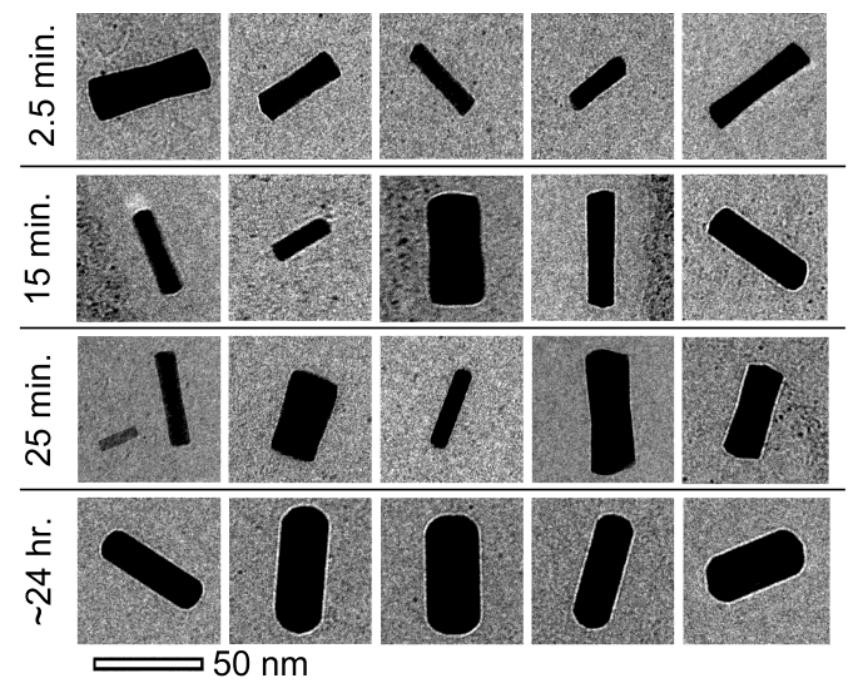

Figure 5. Cryo-TEM images of rods found in nanorod growth solution frozen after a) 2.5 minutes, b) 15 minutes, c) 25 minutes and d) 24 hours, demonstrating examples of the various intermediate geometries found in each sample. Note that rods are still nucleating in the 25 minute sample, as shown by the small, pale rod in the lower left quadrant of the first panel. 
The end profiles of the imaged rods varied from flat-ended through conical to hemispherical as time progressed but their sizes were surprisingly similar. Considering the particle dimensions and geometry observed in the TEM in the context of the spectral data which show steadily increasing optical density (Figure 4) it is clearly not possible for all seeds to grow to nanorods in parallel from the point of seeding. That is, each seed particle does not begin growing into a nanorod immediately. This is supported by fact that the volume percent of metallic gold at 2.5 minutes (Figure 2 ) is only about $1 \%$ that of the final product whereas the volumes of the largest nanoparticles observed at this time are already approximately $50-60 \%$ that of the final shape.

Previous kinetic models ${ }^{29,32}$ for nanorod growth have assumed that the applicable geometry is that of a hemispherically-capped right cylinder. Our cryo-TEM data suggest that this is not strictly correct. Furthermore, the distributions of shapes of the nanorods in an ensemble is also expected to exert an effect on its optical extinction. ${ }^{31}$ The presence of intermediate particle geometries is also evident from optical absorption spectra collected towards the end of the primary reaction prior to ageing (Figure 6). These spectra reveal that the average geometry of the distribution is changing, and that the concentration of shapes with a redshifted longitudinal resonance is decreasing while that of shapes with a more blue-shifted longitudinal resonance is increasing. Since the bulk of the shift in resonance wavelength is associated with the longitudinal peak, and because the volume of $\mathrm{Au}^{0}$ has ceased to change much at this point in the growth process, it can be deduced that the spectral changes are due to the flared end-cap geometry morphing into the final, familiar, hemispherical-cap. This type of shape change has been shown to be associated with a significant blue-shifting of the longitudinal plasmon resonance, even if the aspect ratio itself does not change. ${ }^{31}$ This change is driven by surface energy since the early flared end-cap morphology must have a relatively smaller area of the low energy $\{111\}$ facets than the well-aged rods at the end of the growth 
process. A remaining issue is how the flared end-caps developed in the first place. We suggest that this results from two factors; first the main growth is along a $<100>$ direction thus favoring flattened $\{100\}$ rod ends during that initial time period when rapid growth is dominant, and second, the limited lateral growth on the sides that does occur must proceed somewhat faster on freshly-formed side surfaces than on the older ones. This difference in reactivity of side surfaces with age might be the result of the surface passivating process requiring some time to reach an optimum configuration. Such a situation would naturally result initially in a slightly flared particle, followed later by its conversion later into the familiar hemispherically-capped cylinder.

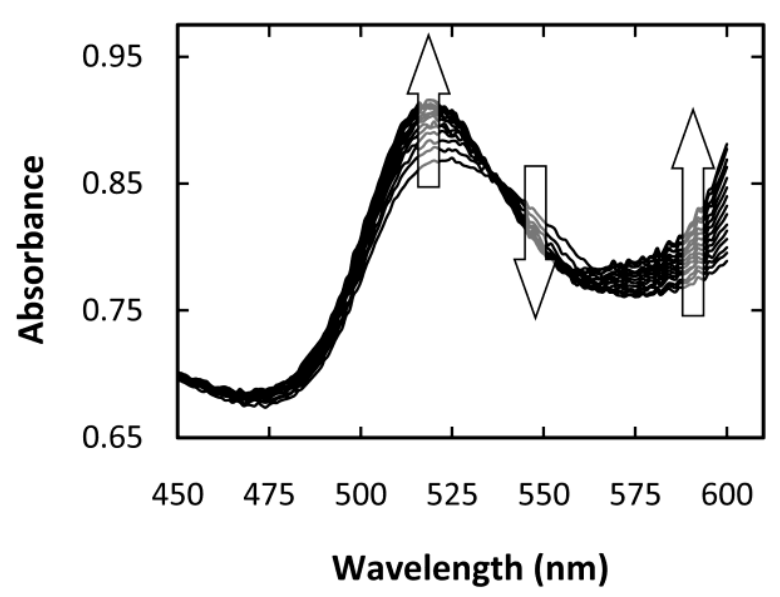

Figure 6. Absorbance spectra collected towards the end of growth sequence $(0.5<t<1$ hour) when a nearly constant volume of $\mathrm{Au}^{0}$ has been achieved. Arrows show change in absorbance with time.

Some insights into the above phenomena may be gained from two simple mathematical models. Using the first model we illustrate the effect of nanorod shape on the optical spectra, while the second model demonstrates how surface energy considerations affect the aspect ratio of rods during the growth process. 
Intermediate nanoparticle geometries can be approximated from the TEM data (e.g. Figure 5) by assuming rotational symmetry about the longitudinal axis. Figure 7 illustrates prototypical examples of these intermediate geometries and the designations that we have given them.

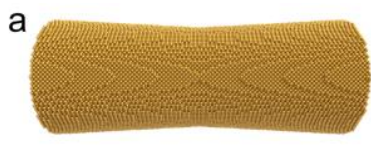

\section{b}

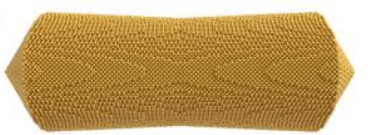

C

d
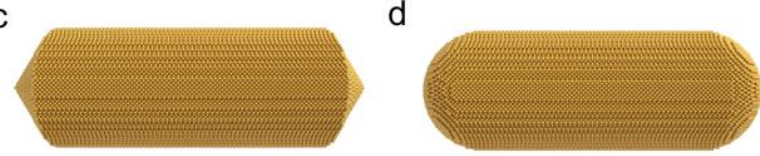

Figure 7. Geometries observed during growth of gold nanorods, (a) bow-tie (BT), (b) conically-capped bow-tie (CBT), (c) conically-capped cylinder (CC) and (d) the final nanorod morphology, a hemispherically-capped cylinder (SC).

Optical extinction spectra, Figure 8, were calculated for ensembles of these prototypical shapes using sizes that were observed in the TEM data. The shapes intermediate between the prototypes were generated by assuming a linear progression of the particle dimensions. It was assumed that the end product in the growth sequence is the familiar spherically capped cylinder (SC). Besides the well-known strong red-shifting associated with an increase in aspect ratio, an increase in volume also has a red-shifting effect on the main extinction peak, while a change in the shape of the end-caps can cause blue- or red-shifts, depending on its nature. The BT particle geometry was assigned as the most primitive (optically resolvable) structure in the growth sequence and so the progression in morphology is $\mathrm{BT} \rightarrow \mathrm{CBT} \rightarrow \mathrm{CC}$ $\rightarrow \mathrm{SC}$ (as indicated by the arrow in Figure 8 ). The sequence of simulations then shows that the longitudinal peak initially blue-shifts, corresponding mainly to an increase in aspect ratio, 
and then red shifts when the waist of the particle begins to develop and/or the rod decreases in length and the end-caps change geometry from conical to hemispherical.

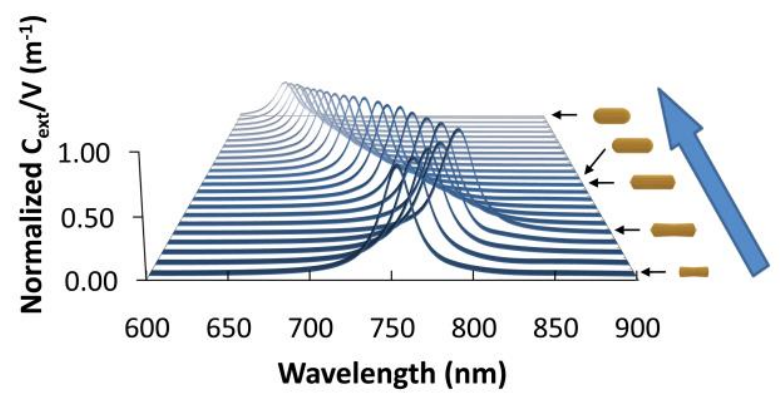

Figure 8. Normalized simulated nanoparticle spectra. Corresponding geometries are indicated on the right with intermediate spectra generated by a linear interpolation of the relevant geometries. The large arrow indicates the direction of development.

This illustrative model reproduces the behavior of the experimentally measured data, Figure 9. However, the experimental data has a somewhat broader longitudinal plasmon resonance peak than the simulated data, presumably due to it having a broader distribution of particle sizes than the simulated data (our simulations aimed to correctly account for peak position rather than for peak broadening). We therefore conclude that the evolution of the experimental optical spectra, and also of the growing nanorods, may be ascribed to the sequence of events shown in Figure 8. 

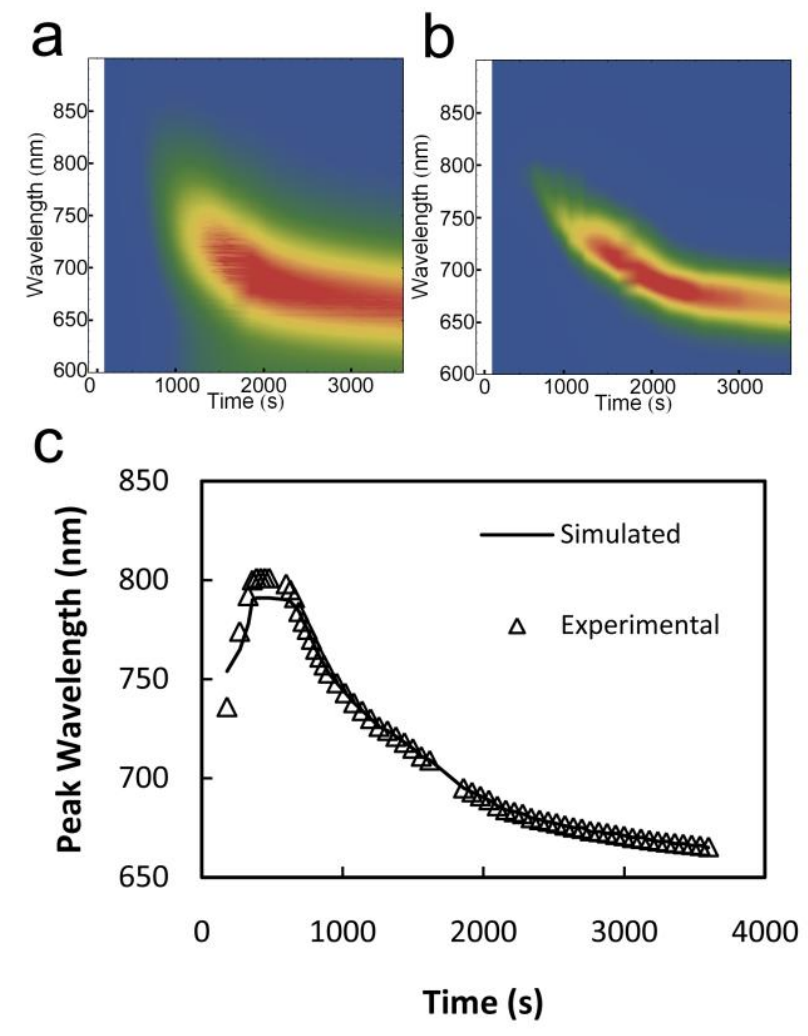

Figure 9. (a) Measured absorbance spectrum of growing nanorod solution with $0.65 \mathrm{mM}$ ascorbic acid. (b) Distributions of calculated particle spectra progressing from BT - CBT CC - SC. Spectra normalized to experimental peak position and gold volume. (c) Longitudinal peak positions of experimental and simulated data from (a) and (b) respectively.

The second mathematical model illustrates the effect of surface energy and kinetics on the aspect ratio of the rod. It begins with the recognition that the development of rod-like shapes in a single crystal of face-centered cubic gold can only result if growth proceeds more rapidly along a particular pair of oppositely aligned $<100>$ directions than any of the other possible directions. While it has been generally argued that growth is restricted in the other directions by a layer of surfactant and/or silver species that passivates the sides of the rod, here we will show that a mechanism based on the interplay between volume and surface energy will also provide rod-like shapes. This scheme does not impose any a priori restrictions for growth rates along different crystallographic directions, and requires only that the surface energies of 
different crystallographic faces differ. It is therefore fundamentally different in nature, for example, to the recently published growth model of Seyed-Razavi et al. in which the growth of gold nanorods was modeled by prohibiting growth on any face other than the $\{100\} .^{57}$

To simplify the development of a model based on surface energies we considered only two kinds of crystal facet, corresponding to either the sides or the end caps of the nanorods, with surface energies of $S_{\text {side }}$ and $S_{\text {cap }}$ respectively. In general, a number of studies have concluded that $S_{\text {side }}<S_{\text {cap }}$ and that the difference may be attributed to factors such as the selective binding of $\mathrm{C}_{16} \mathrm{TABr}^{22,35-36}$ and/or Ag-containing compounds along the sides. ${ }^{15,37}$ Of course, the $\{100\},\{110\}$ or $\{250\}$ facets found along the sides of the rod would have a higher surface energy than the highly coordinated $\{111\}$ facets observed at the ends of well-aged rods in a vacuum environment. However, in the solutions used to make rods this original difference in energy is evidently diminished or even reversed by the selective binding of material derived from the surrounding solution onto the less highly coordinated side facets. The important factor that influences the final shape of a nanorod is the ratio $S=S_{\text {side }} / S_{\text {cap }}$ operating in the aqueous environment during growth. The mechanism by which Ag ions control rod shape can then be readily explained since the presumed attachment of Ag ions or complexes that occurs on the sides of the rod will change the surface energy there and thus the $S_{\text {side }} / S_{\text {cap }}$ ratio. For simplicity, we depict a nanorod as a hemispherically-capped right cylinder of total length, $L$, and diameter, $D$, with a volume, $V$, of gold. (Although the model could be extended to the more complex shapes described above, this would not provide a materially different result and greatly complicates the analytical expressions.)

For convenience, $V$ can be expressed (see Section 6 of Supporting Information) in terms of the aspect ratio, $Y=L / D$, as

$$
V=\pi \frac{D^{3}}{8}\left[\frac{4}{3}+2 \cdot(Y-1)\right]
$$


By analogy to standard homogenous nucleation and growth theory, the work done to create a particular nanorod can be expressed as the difference between its total surface energy and the chemical energy released when the volume $V$ of gold was reduced to $\mathrm{Au}^{0}$ from $\mathrm{Au}^{\mathrm{I}}$.

Normalizing both the surface energy and the volume energy with respect to $S_{\text {cap }}$, and allowing the coefficient $\beta$ to contain the normalized volumetric energy of the nanorod per $\mathrm{nm}^{3}$ (see Supporting Information), the net work done $(\Delta W)$ can be expressed as

$$
\Delta W_{/} / S_{\text {cap }}=4 \pi R^{2}+2 \pi R C \frac{S_{\text {gide }}}{S_{\text {cap }}}-\pi R^{3}\left[\frac{4}{3}+2 \cdot(Y-1)\right] \cdot \beta
$$

$\beta$ was estimated from the observation that $\mathrm{Au}$ spheres of $\sim 5 \mathrm{~nm}$ diameter represent a threshold for longer term thermodynamic stability in growth solutions of the type used here, so that when $R=2.5 \mathrm{~nm}$ and $S \approx 0.5$, for example, $\beta$ must be approximately $1.20 \mathrm{~m}^{-1}$ in order to give $\Delta W / S_{\text {cap }}=0$ (the threshold condition). The trajectory that can be followed by a growing nanoparticle can be determined if (1) it is assumed that changes in shape occur in a manner that lowers its energy, and (2) that a Monte Carlo-driven stochastic process of incremental volume change applies. 

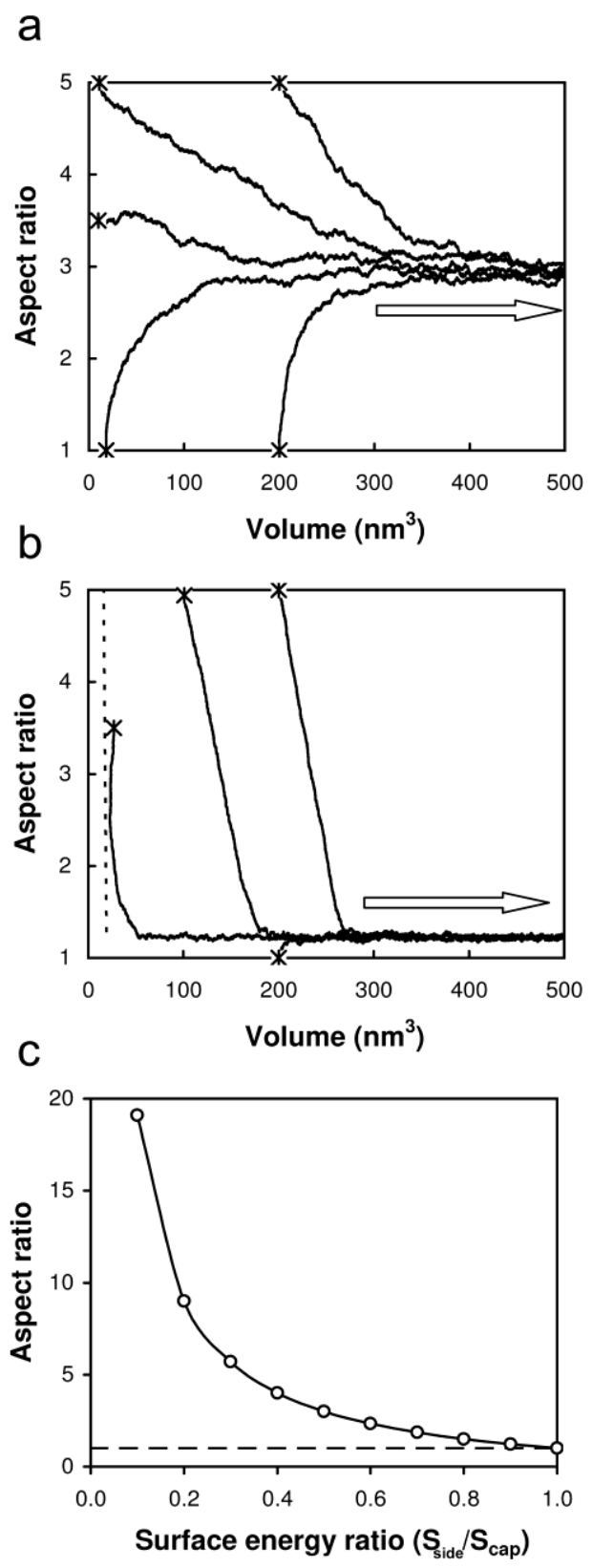

Figure 10. (a) Example of predicted growth trajectories for the case of $S_{\text {side }} / S_{\text {cap }}=0.5$ for five differently-sized seeds that grow stochastically in a way that reduces their overall energy. Starting seed shown with symbol '*'. (b) Example of predicted growth trajectories for case of $S_{\text {side }} / S_{\text {cap }}=0.9$ for four differently-sized seeds that grow stochastically in a way that reduces their overall energy. Spontaneous dissolution of the seed will occur in the region to the left of the dashed line. (c) Effect of ratio $S_{\text {side }} / S_{\text {cap }}$ on the predicted aspect ratio of a hemisphericallycapped right cylindrical nanorod in terms of the simple model proposed. 
Figure 10(a) and (b) shows data from simulations with plots of growth trajectories for several starting seed geometries for the illustrative cases of $S=0.5$ and $S=0.9$. It is clear that growth cannot occur if the seed is too small (dashed line) as in that case only dissolution is energetically possible. However, any seed that is larger than the critical size will grow on a trajectory that reaches the final aspect ratio quite rapidly. Furthermore, the final aspect ratio is completely determined by the value of $S$. In Figure 10(c) we plot the aspect ratios as a function of $S$. It can be seen that the stable aspect ratio of the particle can be varied from 1.0 (for $S=1$ ) to greater than 19 (for $S=0.2$ ). However, given that real gold nanorods are generally found to have aspect ratios in the 2 to 5 range, it follows that this model predicts that $0.35<S<0.65$ in typical growth solutions.

\section{DISCUSSION}

To account for the difference in the amount of gold deduced from the optical absorbance versus that implied from the particle dimensions observed using TEM, both the number and volume of nanorods in the solution must be increasing with time. This would imply both that there is a threshold condition for a seed particle which must be satisfied before it will begin growing toward the nanorod geometry, and that this threshold is reached at different times in individual seed particles. Furthermore, an analysis of the products of nanorod syntheses provides evidence for a bifurcation in particle dimensions at $\sim 5 \mathrm{~nm} .{ }^{58}$ This bifurcation suggests that if the seed particle does not undergo the symmetry-breaking event before it has reached about $5 \mathrm{~nm}$ in diameter, then it will never form a nanorod. Instead such a nanoparticle will coarsen to become one of the larger spherical nanoparticles that are inevitably present in small proportions in the ensembles produced by the seeded-growth technique. However, if the symmetry-breaking threshold condition is met while it is still smaller than the $5 \mathrm{~nm}$ threshold then anisotropic growth is initiated and the resulting 
nanorods achieve a reasonably tight dispersion of aspect ratios. Once the threshold condition for rod-growth has been met the initial growth rate is very rapid. The initial mechanism is analogous to a 'popcorn-like' event followed by a gradual further development of the particle's dimensions. A possible explanation for the failure of seeds larger than $5 \mathrm{~nm}$ to form nanorods may be found in the 'nanophase stability diagrams' of Barnard et al. ${ }^{59}$ which show that a gold nanoparticle at room temperature will be most stable when adopting the icosohedral form for a diameter less than about $4 \mathrm{~nm}$, and decahedral when the diameter is above $4 \mathrm{~nm}$ but less than about $15 \mathrm{~nm}$, Figure 11 . The implication here for the growth of rods is that only icosohedral particles can seed a nanorod so any spherical seed that coarsens past the critical diameter of about $5 \mathrm{~nm}$ will not contribute to rod growth and will instead coarsen to an equiaxed decahedral or fcc shape.

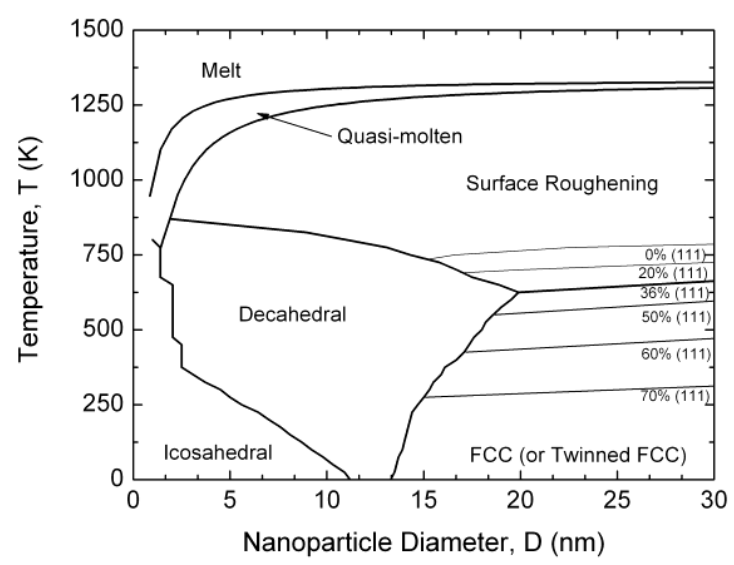

Figure 11. Calculated phase stability diagram for spherical gold nanoparticles. Reprinted with permission from ref ${ }^{59}$. Copyright 2009 American Chemical Society.

While the threshold condition that initiates rod growth could be crystallographic, the idea of electric field assisted growth ${ }^{19}$ could also provide an explanation. The origin of this electric field would be the adsorption of ionic species at the surface of the seed particles 
where fluctuations in the charge density or local concentration of $\mathrm{Au}$ ions could induce a symmetry-breaking event. In either event, the random fluctuations in local environment that trigger the symmetry-breaking threshold require some time to occur, hence the stochastic delay in growth of the rod. This effect would be more significant for smaller particles leading to another potential reason for the bifurcation in particle growth trajectories at about $5 \mathrm{~nm}$ diameter. There is no reason why stochastic growth of this type should be restricted to gold nanorods, and evidence of its occurrence in the growth of other anisotropic nanoparticles should be sought.

\section{CONCLUSION}

Whereas previous work has assumed a gradual and parallel growth of seeds into nanorods, here we have shown that intermediate geometries are formed by a comparatively early growth event which is of a stochastic nature. These intermediate particle geometries have been observed in cryo-TEM data and inferred from optical extinction data. The transformation between these intermediate geometries has been simulated and the associated optical properties calculated, and the results are consistent with the experimental data. The important influence of the ratio of surface energies between side and cap of the nanorod was identified. The reduction mechanism of gold species was also considered. The concentration of gold in the nanorod product is closely related to the expected amount from the disproportionation mechanism although the formation of an Au-ascorbate complex as intermediate is not excluded. The blue-shifting of the longitudinal plasmon peak that occurs at the end of the growth process is associated with a change in shape from 'bow tie' to hemispherically-capped right-cylinder. 


\section{METHODS}

The following chemicals were purchased commercially and used as received: $\mathrm{C}_{16} \mathrm{TABr}$ and ascorbic acid (Alfa Aesar), $\mathrm{HAuBr}_{4}, \mathrm{AgNO}_{3}$ and $\mathrm{KBH}_{4}$ (Sigma Aldrich), and $\mathrm{C}_{16} \mathrm{TACl}$ (Nanjing Robiot). Tetrachloroauric acid $\left(\mathrm{HAuCl}_{4}\right)$ was synthesized according to published methods. ${ }^{60}$ Water was purified by a MilliQ system $(18.2 \mathrm{~m} \Omega)$.

Gold nanorods were prepared using a variation of a reported method. ${ }^{14,}{ }^{36}$ First, growth solutions (each of $10 \mathrm{~mL}$ volume) with the following concentrations were prepared: $0.1 \mathrm{M} \mathrm{C}_{16} \mathrm{TABr}, 0.5 \mathrm{mM}$ tetrachloroauric acid $\left(\mathrm{HAuCl}_{4}\right), x \mathrm{mM}$ ascorbic acid (where $x$ varied from 0.4 to 1.0 ) and 50,70 or 100 $\mu \mathrm{M}$ silver nitrate $\left(\mathrm{AgNO}_{3}\right)$. Separately, a seed solution was prepared by adding $0.5 \mathrm{~mL}$ of ice-cold $0.01 \mathrm{M}$ potassium borohydride to $10 \mathrm{~mL}$ of a solution containing $\mathrm{HAuCl}_{4}(0.1 \mathrm{mM})$ and $\mathrm{C}_{16} \mathrm{TABr}(25$ $\mathrm{mM})$. The seed solution was aged for one hour at $30^{\circ} \mathrm{C}$. Growth was initiated by addition of $10 \mu \mathrm{L}$ of the seed solution to the a growth solution and the mixture was maintained at $30^{\circ} \mathrm{C}$ for the duration of the reaction.

UV-Vis-NIR spectra were recorded using an Agilent 8453E photodiode array spectrophotometer. During the early stages of rod growth (up to $5-10 \mathrm{~min}$., depending on the ascorbic acid concentration), the optical density of the suspension was quite low and so a $10 \mathrm{~cm}$ path length quartz cuvette (Starna) was utilized during this phase. For growth times longer than $10 \mathrm{~min}$., a $1 \mathrm{~cm}$ quartz cuvette was used. Kinetics data for the $0.65 \mathrm{mM}$ ascorbic acid sample approached the detection limit of the spectrophotometer towards the end of the reaction so it was diluted for the remainder of the reaction. The gold concentration in the $0.65 \mathrm{mM}$ sample follows the same curve shape as that of the $0.60 \mathrm{mM}$ ascorbic acid sample and so diluting the sample does not appear to have affected this stage of the reaction significantly.

The concentration of $\mathrm{Au}^{0}$ during the reactions was determined by monitoring the optical absorbance of solutions at $390 \mathrm{~nm}$. Metallic gold exhibits an optical response that is relatively independent of shape in the wavelength range 350 to $450 \mathrm{~nm}$, which lies within the region of interband transitions. ${ }^{61-}$ ${ }^{62}$ In this range the absorbance depends predominantly on the number and volume of $\mathrm{Au}^{0}$ nanoparticles present and . Therefore, this has been used in the past to provide an estimate of $\mathrm{Au}^{0}$ 
concentration. ${ }^{25,51,63-64}$ In the present work, the method was calibrated using well-aged suspensions of $\mathrm{C}_{16} \mathrm{TABr}$-stabilized gold nanoparticles of known concentration that were well-aged. This was to ensure such that all gold was reduced and that size effects did not contribute to the absorbance at 390 $\mathrm{nm}$. Another verification of the technique could be found by correlating the published inductively coupled plasma-mass spectrometry (ICP-MS) and optical measurements of Orendorff et al. ${ }^{65}$ for gold nanorods (see Section 2 of Supporting Information). The optical method provides an estimate of $\mathrm{Au}^{0}$ that is within $20 \%$ of the ICP-MS result, a discrepancy that is much smaller than the $50 \%$ loss of $\mathrm{Au}$ found by Orendorff et al. in their overall mass balance. The discrepancy in the original work may have been due to some of the $\mathrm{Au}$ remaining in the oxidized state, or due to some of the $\mathrm{Au}^{0}$ not reporting to the pellet that was analyzed, but the net result is that optical and ICP-MS analyses are considerably more accurate than the overall level of uncertainty of the reacting system.

Cryo-TEM experiments were performed to probe the early stages of development of gold nanorods. The rods were synthesized in the sample preparation room adjacent to the microscope. Our standard technique, which was found in prior work to produce a colloid with a final absorption peak at about $680 \mathrm{~nm}$ was applied. However, the position of this peak was not re-verified during the sample preparation as a suitable spectrometer was not available at the location of the TEM. Images were taken using an FEI TECNAI F30 G2 TEM at $300 \mathrm{keV}$ using a sample stage cooled with liquid nitrogen. For the sample preparation no chemical changes were made to the standard growth solution and the concentrations and method were identical to those used in the kinetics studies. TEM samples were prepared with the aid of an FEI Vitrobot system to ensure reproducible sample conditions. In this step 2 to $3 \mu \mathrm{L}$ of sample was taken and placed on a TEM grid held in a controlled environment at $30^{\circ} \mathrm{C}$ and $100 \%$ relative humidity. A blotting time of $8 \mathrm{~s}$ was used to achieve an ice thickness of $\sim 50-$ $100 \mathrm{~nm}$. After blotting, the TEM grid was plunged into a bath of liquid ethane to form a layer of vitreous ice. Multiple grids were prepared and subsequently stored in liquid nitrogen. Any failure of the specimen preparation process, as for example could hypothetically occur by reheating of the sample, necessarily renders the TEM samples unusable. Therefore, the observed rod structures may be confidently assumed to have grown prior to freezing 
The optical extinction properties of gold nanorods of various shapes were calculated using the DDSCAT code of Draine and Flatau. ${ }^{66-67}$ This method uses the discrete dipole approximation (DDA) and has been found in previous work to be accurate provided that the dipole spacing is sufficiently small. ${ }^{68}$ A dipole spacing of between 0.4 and $0.6 \mathrm{~nm}$ was used here, with the larger spacing used for the larger rods. The target was sampled once in the longitudinal direction and twice in the transverse direction to give an average optical extinction representative of a colloidal suspension.

Acknowledgment. This work was supported by the Australian Research Council and AGR Matthey Limited. We also acknowledge the technical, scientific and financial assistance from the Australian Microscopy \& Microanalysis Research Facility (AMMRF) for funding cryoTEM studies at the University of Queensland.

Supporting Information. Application of an empirical model for growth of nanorods using coupled equations of Henkel et al. UV-visible spectra showing the identity of the soluble Au species. Explanation of the calibration of content of metallic gold to optical extinction spectra. Optical extinction spectra showing the effect of increasing concentration of ascorbic acid on the rod-forming reaction. TEM image of frozen growth solution. Principles used to drive shape change in authors' mathematical model of evolving geometry. Derivation of algebraic expression for net work done to form a nanorod. This material is available free of charge via the Internet at http://pubs.acs.org.

\section{REFERENCES}

1. Edgar, J. A.; Cortie, M. B. Nanotechnological Applications of Gold. In Gold. Science and Applications; Corti, C.; Holliday, R., Eds.; Taylor and Francis: London, 2009; 369-397.

2. Pérez-Juste, J.; Mulvaney, P.; Liz-Marzán, L. M., Patterning and Encryption Using Gold Nanoparticles. Int. J. Nanotechnol. 2007, 4, 215 - 224. 
3. Pissuwan, D.; Valenzuela, S. M.; Cortie, M. B., Prospects for Gold Nanorod Particles in Diagnostic and Therapeutic Applications. Biotechnol. Genet. Eng. Rev. 2008, 25, 93-112.

4. Stokes, N. L.; Edgar, J. A.; McDonagh, A. M.; Cortie, M. B., Spectrally Selective Coatings of Gold Nanorods on Architectural Glass. J. Nanopart. Res. 2010, 12, 2821 - 2830.

5. Zijlstra, P.; Chon, J. W. M.; Gu, M., Five-Dimensional Optical Recording Mediated by Surface Plasmons in Gold Nanorods. Nature 2009, 459, 410-413.

6. Murphy, C. J.; Gole, A. M.; Hunyadi, S. E.; Orendorff, C. J., One-Dimensional Colloidal Gold and Silver Nanostructures. Inorg. Chem. 2006, 45, 7544-7554.

7. Perez-Juste, J.; Pastoriza-Santos, I.; Liz-Marzan, L. M.; Mulvaney, P., Gold Nanorods: Synthesis, Characterization and Applications. Coord. Chem. Rev. 2005, 249, $1870-1901$.

8. Yu, Y.-Y.; Chang, S.-S.; Lee, C.-L.; Wang, C. R. C., Gold Nanorods: Electrochemical Synthesis and Optical Properties. J. Phys. Chem. B 1997, 101, 6661-6664.

9. Hirsch, L. R.; Stafford, R. J.; Bankson, J. A.; Sershen, S. R.; Rivera, B.; Price, R. E.; Hazle, J. D.; Halas, N. J.; West, J. L., Nanoshell-Mediated Near-Infrared Thermal Therapy of Tumors under Magnetic Resonance Guidance. Proc. Natl. Acad. Sci. U. S. A. 2003, 100, 13549-13554.

10. Loo, C.; Lowery, A.; Halas, N.; West, J.; Drezek, R., Immunotargeted Nanoshells for Integrated Cancer Imaging and Therapy. Nano Lett. 2005, 5, 709-711.

11. Oldenburg, S. J.; Averitt, R. D.; Westcott, S. L.; Halas, N. J., Nanoengineering of Optical Resonances. Chem. Phys. Lett. 1998, 288, 243-247. 
12. Harris, N.; Ford, M. J.; Mulvaney, P.; Cortie, M. B., Tunable Infrared Absorption by Metal Nanoparticles: The Case for Gold Rods and Shells. Gold Bull. 2008, 41, 5-14.

13. Jain, P. K.; Lee, K. S.; El-Sayed, I. H.; El-Sayed, M. A., Calculated Absorption and Scattering Properties of Gold Nanoparticles of Different Size, Shape, and Composition: Applications in Biological Imaging and Biomedicine. J. Phys. Chem. B. 2006, 110, 72387248

14. Jana, N. R.; Gearheart, L.; Murphy, C. J., Seed-Mediated Growth Approach for Shape Controlled Synthesis of Spheroidal and Rod-Like Gold Nanoparticles Using a Surfactant Template. Adv. Mater. 2001, 13, 1389-1393.

15. Hubert, F.; Testard, F.; Spalla, O., Cetyltrimethylammonium Bromide Silver Bromide Complex as the Capping Agent of Gold Nanorods. Langmuir 2008, 24, 9219-9222.

16. Jana, N. R., Gram-Scale Synthesis of Soluble, Near-Monodisperse Gold Nanorods and Other Anisotropic Nanoparticles. Small 2005, 1, 875-882.

17. Zijlstra, P.; Bullen, C.; Chon, J. W. M.; Gu, M., High-Temperature Seedless Synthesis of Gold Nanorods. J. Phys. Chem. B 2006, 110, 19315-19318.

18. Busbee, B. D.; Obare, S. O.; Murphy, C. J., An Improved Synthesis of High-AspectRatio Gold Nanorods. Adv. Mater. 2003, 15, 414-416.

19. Perez-Juste, J.; Liz-Marzan, L. M.; Carnie, S.; Y.C.Chan, D.; Mulvaney, P., ElectricField-Directed Growth of Gold Nanorods in Aqueous Surfactant Solutions. Adv. Funct. Mater. 2004, 14, 571-579. 
20. Tornblom, M.; Henriksson, U., Effect of Solubilization of Aliphatic Hydrocarbons on Size and Shape of Rodlike C(16)TABr Micelles Studied by H-2 NMR Relaxation. J. Phys. Chem. B 1997, 101, 6028-6035.

21. Aswal, V. K.; Goyal, P. S.; Amenitsch, H.; Bernstorff, S., Counterion Condensation in Ionic Micelles as Studied by a Combined Use of SANS and SAXS. Pramana: J. Physics 2004, 63, 333-338.

22. Gao, J.; Bender, C. M.; Murphy, C. J., Dependence of the Gold Nanorod Aspect Ratio on the Nature of the Directing Surfactant in Aqueous Solution. Langmuir 2003, 19, 9065 9070.

23. Jana, N. R.; Gearheart, L. A.; Murphy, C. J., Evidence for Seed-Mediated Nucleation in the Chemical Reduction of Gold Salts to Gold Nanoparticles. Chem. Mater. 2001, 13, $2313-2322$.

24. Liu, M.; Guyot-Sionnest, P., Mechanism of Silver(I)-Assisted Growth of Gold Nanorods and Bipyramids. Journal of Phys. Chem. B 2005, 109, 22192-22200.

25. Sau, T. K.; Murphy, C. J., Seeded High Yield Synthesis of Short Au Nanorods in Aqueous Solution. Langmuir 2004, 20, 6414-6420.

26. Zweifel, D. A.; Wei, A., Sulfide-Arrested Growth of Gold Nanorods. Chem. Mater. 2005, 17, 4256-4261.

27. Niidome, Y.; Nishioka, K.; Kawasaki, H.; Yamada, S., Rapid Synthesis of Gold Nanorods by the Combination of Chemical Reduction and Photoirradiation Processes; Morphological Changes Depending on the Growing Processes. Chem. Comm. 2003, 18, $2376-2377$ 
28. Becker, J.; Schubert, O.; Sonnichsen, C., Gold Nanoparticle Growth Monitored in Situ Using a Novel Fast Optical Single-Particle Spectroscopy Method. Nano Lett. 2007, 7, 16641669.

29. Gulati, A.; Liao, H.; Hafner, J. H., Monitoring Gold Nanorod Synthesis by Localized Surface Plasmon Resonance. J. Phys. Chem. B. 2006, 110, 22323 - 22327.

30. Prescott, S. W.; Mulvaney, P., Gold Nanorod Extinction Spectra. J. of Applied Physics 2006, 99, 123504.

31. Xu, X.; Cortie, M. B., Shape Change and Color Gamut in Gold Nanorods, Dumbbells and Dog-Bones. Adv. Funct. Mater. 2006, 16, 2170-2176.

32. Henkel, A.; Schubert, O.; Plech, A.; Sonnichsen, C., Growth Kinetic of a Rod-Shaped Metal Nanocrystal. J. Phys. Chem. C 2009, 113, 10390-10394.

33. Morita, T.; Tanaka, E.; Inagaki, Y.; Hotta, H.; Shingai, R.; Hatakeyama, Y.; Nishikawa, K.; Murai, H.; Nakano, H.; Hino, K., Aspect-Ratio Dependence on Formation Process of Gold Nanorods Studied by Time-Resolved Distance Distribution Functions. $J$. Phys. Chem. C 2010, 114, 3804-3810.

34. Brioude, A.; Jiang, X. C.; Pileni, M. P., Optical Properties of Gold Nanorods: DDA Simulations Supported by Experiments. J. Phys. Chem. B 2005, 109, 13138-13142.

35. Nikoobakht, B.; El-Sayed, M. A., Evidence for Bilayer Assembly of Cationic Surfactants on the Surface of Gold Nanorods. Langmuir 2001, 17, 6368-6374.

36. Nikoobakht, B.; El-Sayed, M. A., Preparation and Growth Mechanism of Gold Nanorods (NRs) Using Seed-Mediated Growth Method. Chem. Mater. 2003, 15, 1957-1962. 
37. Niidome, Y.; Nakamura, Y.; Honda, K.; Akiyama, Y.; Nishioka, K.; Kawasaki, H.; Nakashima, N., Characterization of Silver Ions Adsorbed on Gold Nanorods: Surface Analysis by Using Surface-Assisted Laser Desorption/ionization Time-of-flight Mass Spectrometry. Chem. Comm. 2009, 1754-1756.

38. Carbó-Argibay, E.; Rodróguez-González, B.; Gómez-Graña, S.; Guerrero-Martínez, A.; Pastoriza-Santos, I.; Pérez-Juste, J.; Liz-Marzán, L. M., The Crystalline Structure of Gold Nanorods Revisited: Evidence for Higher-Index Lateral Facets. Angew. Chem. Int. Ed. 2010, 49, 9397-9400.

39. Murphy, C. J.; Sau, T. K.; Gole, A. M.; Orendorff, C. J.; Gao, J.; Gou, L.; Hunyadi, S. E.; Li, T., Anisotropic Metal Nanoparticles: Synthesis, Assembly, and Optical Applications. J. Phys. Chem. B 2005, 109, 13857-13870.

40. Kawasaki, H.; Nishimura, K.; Arakawa, R., Influence of the Counterions of Cetyltrimetylammonium Salts on the Surfactant Adsorption onto Gold surfaces and the Formation of Gold Nanoparticles. J. Phys. Chem. C 2007, 111, 2683-2690.

41. Miranda, O. R.; Ahmadi, T. S., Effects of Intensity and Energy of CW UV Light on the Growth of Gold Nanorods. J. Phys. Chem. B 2005, 109, 15724-15734.

42. Placido, T.; Comparelli, R.; Giannici, F.; Cozzoli, P. D.; Capitani, G.; Striccoli, M.; Agostiano, A.; Curri, M. L., Photochemical Synthesis of Water-Soluble Gold Nanorods: The Role of Silver in Assisting Anisotropic Growth. Chem. Mater. 2009, 21, 4192-4202.

43. Abdelmoti, L. G.; Zamborini, F. P., Potential-Controlled Electrochemical SeedMediated Growth of Gold Nanorods Directly on Electrode Surfaces. Langmuir 2010, 26, 13511-13521. 
44. Sepúlveda, L.; Cortés, J., Ionization Degrees and Critical Micelle Concentrations of Hexadecyltrimethylammonium and Tetradecyltrimethylammonium Micelles with Different Counterions. J. Phys. Chem. 1985, 89, 5322 - 5324.

45. Attwood, D.; Florence, A. T., Surfactant Systems : Their Chemistry, Pharmacy and Biology. Chapman and Hall: London, 1983; p 84,.

46. Zümreoglu-Karan, B., A Rationale on the Role of Intermediate Au(III)-Vitamin C Complexation in the Production of Gold Nanoparticles. J. Nanopart. Res. 2009, 11, 10991105

47. Gou, L.; Murphy, C. J., Fine-Tuning the Shape of Gold Nanorods. Chem. Mater. 2005, 17, 3668-3672.

48. Jaiswal, P. V.; Ijeri, V. S.; Srivastava, A. K., Effect of Surfactants on the Dissociation Constants of Ascorbic and Maleic Acids. Colloid. Surf. B 2005, 46, 45 - 51.

49. Evans, D. H.; Lingane, J. J., Standard Potentials of the Couples Involving $\mathrm{AuBr}_{4}{ }^{-}$ ,AuBr${ }_{2}^{-}$and $\mathrm{Au}$ in Bromide Media. J. Electroanal. Chem. 1963, 6, 1 - 10.

50. Gammons, C. H.; Yu, Y.; Williams-Jones, A. E., The Disproportionation of Gold (I) Chloride Complexes at 25 to $200^{\circ}$ C. Geochim. Cosmochim. Ac. 1997, 61, $1971-1983$.

51. Rodriguez-Fernandez, J.; Perez-Juste, J.; Mulvaney, P.; Liz-Marzan, L. M., SpatiallyDirected Oxidation of Gold Nanoparticles by Au(III)-CTAB Complexes. J. Phys. Chem. B. 2005, 109, 14257-14261.

52. Alekseeva, A. V.; Bogatyrev, V. A.; Dykman, L. A.; Khlebtsov, B. N.; Trachuk, L. A.; Melnikov, A. G.; Khlebtsov, N. G., Preparation and Optical Scattering Characterization 
of Gold Nanorods and Their Application to a Dot-Immunogold Assay. Applied Optics 2005, $44,6285-6295$.

53. Boleininger, J.; Kurz, A.; Reuss, V.; Sönnichsen, C., Microfluidic Continuous Flow Synthesis of Rod-Shaped Gold and Silver Nanocrystals. Phys. Chem. Chem. Phys. 2006, 8, $3824-3827$.

54. Keul, H. A.; Moller, M.; Bockstaller, M. R., Structural Evolution of Gold Nanorods During Controlled Secondary Growth. Langmuir 2007, 23, 10307-10315.

55. Kou, X. S.; Zhang, S. Z.; Tsung, C. K.; Yeung, M. H.; Shi, Q. H.; Stucky, G. D.; Sun, L. D.; Wang, J. F.; Yan, C. H., Growth of Gold Nanorods and Bipyramids Using CTEAB Surfactant. J. Phys. Chem. B 2006, 110, 16377-16383.

56. Miranda, O. R.; Dollahon, N. R.; Ahmadi, T. S., Critical Concentrations and Role of Ascorbic Acid (Vitamin C) in the Crystallization of Gold Nanorods Within Hexadecyltrimethyl Ammonium Bromide (CTAB)/Tetraoctyl Ammonium Bromide (TOAB) Micelles. Cryst. Growth Design 2006, 6, 2747-2753.

57. Seyed-Razavi, A.; Snook, I. K.; Barnard, A. S., Surface Area Limited Model for Predicting Anisotropic Coarsening of Faceted Nanoparticles. Cryst. Growth Des. 2011, 11, $158-165$.

58. Hubert, F.; Testard, F.; Rizza, G.; Spalla, O., Nanorods Versus Nanospheres: A Bifurcation Mechanism Revealed by Principal Component TEM Analysis. Langmuir 2010, $26,6887-6891$.

59. Barnard, A. S.; Young, N. P.; Kirkland, A. I.; Huis, M. A. v.; Xu, H., Nanogold: A Quantitative Phase Map. ACS Nano 2009, 3, 1431-1436. 
60. Breitinger, D.; Herrmann, W., Synthetic Methods of Organometallic and Inorganic Chemistry. George Thieme Verlag.: Stuttgart, 1999; Vol. 5.

61. Quinten, M., Optical Effects Associated with Aggregates of Clusters. J. Cluster Sci. 1999, $10,321-358$.

62. Ung, T.; Liz-Marzan, L. M.; Mulvaney, P., Gold Nanoparticle Thin Films. Colloids Surf., $A$ 2002, 202, 119-126.

63. Rao, P.; Doremus, R., Kinetics of Growth of Nanosized Gold Clusters in Glass. $J$. Non-Cryst. Solids 1996, 203, 202 - 205.

64. Bohren, C. F.; Huffman, D. R., Absorption and Scattering of Light by Small Particles. John Wiley and Sons: 1983; p 530.

65. Orendorff, C. J.; Murphy, C. J., Quantitation of Metal Content in the Silver-Assisted Growth of Gold Nanorods. J. Phys. Chem. B. 2006, 110, 3990-3994.

66. Draine, B. T.; Flatau, P. J., Discrete-Dipole Approximation for Scattering Calculations. J. Opt. Soc. Am. A 1994, 11, 1491-1499.

67. Draine, B. T.; Flatau, P. J. User Guide for the Discrete Dipole Approximation Code DDSCAT 7.0 2008. http://arxiv.org/abs/0809.0337 (accessed September 2008).

68. Myroshnychenko, V.; Rodríguez-Fernández, J.; Pastoriza-Santos, I.; Funston, A. M.; Novo, C.; Mulvaney, P.; Liz-Marzán, L. M.; Javier-García-de-Abajo, Modelling the Optical Response of Gold Nanoparticles. Chem. Soc. Rev. 2008, 37, 1792 - 1805. 